\title{
Certain approximation properties of Brenke polynomials using Jakimovski-Leviatan operators
}

\author{
Shahid Ahmad Wani' ${ }^{1}$ M. Mursaleen ${ }^{2,3^{*}}$ (D) and Kottakkaran Sooppy Nisar ${ }^{4}$
}

\section{"Correspondence:}

mursaleenm@gmail.com

${ }^{2}$ Department of Medical Research, China Medical University Hospital,

China Medical University (Taiwan), Taichung, Taiwan

${ }^{3} \mathrm{Al}-$ Qaryah, Doharra, Street No. 1 (West), Aligarh, UP, 202002, India

Full list of author information is available at the end of the article

\begin{abstract}
In this article, we establish the approximation by Durrmeyer type Jakimovski-Leviatan operators involving the Brenke type polynomials. The positive linear operators are constructed for the Brenke polynomials, and thus approximation properties for these polynomials are obtained. The order of convergence and the weighted approximation are also considered. Finally, the Voronovskaya type theorem is demonstrated for some particular case of these polynomials.
\end{abstract}

MSC: 40A30; 41A10; 41A25; 41A36

Keywords: Szasz operators; Brenke type polynomials; Jakimovski-Leviatan operators; Modulus of continuity; Weighted space

\section{Introduction and preliminaries}

The Korovkin approximation process plays a crucial role in a wide variety of problems in measure theory, functional analysis, probability theory, and partial differential equations. Korovkin [11] established a well-known simple criterion to decide whether a given sequence $\left(K_{n}\right)_{n \in \mathbb{N}}$ of positive linear operators on the space $C[0,1]$ is an approximation process, i.e., $K_{n}(f) \rightarrow f$ uniformly on $[0,1]$ for every $f \in C[0,1]$. Taking into account this significant result, mathematicians all across the globe have extended this theorem named after Korovkin to other abstract spaces, such as Banach spaces, lattices, algebras, etc. The work of Korovkin laid a foundation and basis for a new theory, mainly referred to as Korovkintype approximation theory.

Favard and Szasz [16] introduced the following example of positive linear operator:

$$
S_{m}(g ; u):=e^{-m u} \sum_{k=0}^{\infty} \frac{(m u)^{k}}{k !} g\left(\frac{k}{m}\right)
$$

where $0 \leq u$ and $g \in \mathbb{C}[0, \infty)$. Numerous mathematicians dealt with the extension of these operators. For example, operators including generalized Appell polynomials [7], Stancu type Baskakov-Durrmeyer operators [10], Stancu type Dunkl generalization of SzászKantorovich operators [13].

(c) The Author(s) 2021. This article is licensed under a Creative Commons Attribution 4.0 International License, which permits use, sharing, adaptation, distribution and reproduction in any medium or format, as long as you give appropriate credit to the original author(s) and the source, provide a link to the Creative Commons licence, and indicate if changes were made. The images or other third party material in this article are included in the article's Creative Commons licence, unless indicated otherwise in a credit line to the material. If material is not included in the article's Creative Commons licence and your intended use is not permitted by statutory regulation or exceeds the permitted use, you will need to obtain permission directly from the copyright holder. To view a copy of this licence, visit http://creativecommons.org/licenses/by/4.0/. 
Leviatan and Jakimovski [8] established the following generalization:

$$
Q_{l}(g ; y):=\frac{e^{-l y}}{h(1)} \sum_{k=0}^{\infty} q_{k}(l y) g\left(\frac{k}{l}\right)
$$

of expression (1.1) by considering the Appell polynomials $p_{k}(x), k \geq 0$ :

$$
h(v) e^{v y}=\sum_{k=0}^{\infty} q_{k}(y) v^{k}
$$

where an analytic function $h(v)=\sum_{l=0}^{\infty} h_{l} v^{l} ;|v|<R, R>1$, and $0 \neq g(1)$, and derived the approximation properties for these above operators.

Recently, Ali Karaisa [9] developed the Durrmeyer-Jakimovski-Leviatan operators of Appell polynomials $p_{k}(y), k \geq 0, \forall h$ on $[0, \infty)$ as follows:

$$
L_{m}(h ; y)=\frac{\exp (-m y)}{g(1)} \sum_{k=0}^{\infty} \frac{p_{k}(m y)}{\mathbb{B}(m+1, k)} \int_{0}^{\infty} \frac{t^{k-1}}{(1+t)^{m+k+1}} h(t) d t, \quad y \geq o,
$$

where beta function $\mathbb{B}(k+1, m)$ is a given by

$$
\begin{aligned}
\mathbb{B}(\alpha, \beta) & =\int_{0}^{\infty} \frac{w^{\alpha-1}}{(1+w)^{\alpha+\beta}} d w \\
& =\frac{\Gamma(\alpha) \Gamma(\beta)}{\Gamma(\alpha+\beta)}, \quad \alpha, \beta \geq 0 .
\end{aligned}
$$

For related work on Jakimovski-Leviatan-beta type integral operators, we refer to [1]. The Szász operators involving Brenke type polynomials were studied in [17].

\section{Construction of operators}

Motivated by the work of Ali Karaisa [9], here we develop the positive linear operators containing the Brenke polynomials $[2,6]$, which possess generating relation of the form

$$
\sum_{k=0}^{\infty} p_{k}(y) t^{k}=g(t) B(y t)
$$

where analytic functions $g$ and $B$ are given by

$$
\begin{aligned}
& \sum_{r=0}^{\infty} g_{r} t^{r}=g(t), \quad g_{0} \neq 0, \\
& \sum_{r=0}^{\infty} b_{r} t^{r}=g(t), \quad b_{r} \neq 0(r \geq 0)
\end{aligned}
$$

and possess explicit expansions:

$$
\sum_{r=0}^{k} g_{k-r} b_{r} y^{r}=p_{k}(y), \quad k=0,1,2, \ldots
$$


Restraining ourselves to $p_{k}(y)$, i.e., the Brenke polynomials satisfying:

(i) $\quad 0 \neq g(1), \quad 0 \leq \frac{g_{k-r} b_{r}}{g(1)}, \quad 0 \leq r \leq k, k=0,1,2, \ldots$,

(ii) $\quad B:[0, \infty) \rightarrow(0, \infty)$,

(iii) generating function (2.1) and power series (2.2) and (2.3) converge for $|t|<R(R>1)$.

Further, the positive linear operators involving $p_{k}(y)$ polynomials are introduced while keeping in consideration the above restrictions by the following manner:

$$
\mathbb{T}_{n}(f ; y):=\frac{1}{g(1) B(n y)} \sum_{k=0}^{\infty} \frac{p_{k}(n y)}{B(n+1, k)} \int_{0}^{\infty} \frac{t^{k-1}}{(1+t)^{n+k+1}} f(t) d t
$$

where $y \geq 0$ and $n \in \mathbb{N}$.

Remark 2.1 For $B(t)=e^{t}$, expressions (2.6) and (2.1) reduce to the expressions represented by (1.4) and (1.2).

\section{Approximation properties of $\mathbb{T}_{n}$ operators}

Korovkin $[11,12]$ derived the results concerning the convergence of sequences $\left(K_{m}(g, y)\right)_{m=1}^{\infty}$, where $K_{m}(g, y)$ are positive linear operators. For instance, if $K_{m}(g, y)$ uniformly converges to $g$ for some particular cases $1, t, t^{2} \equiv g(t)$, likewise it performs such activity for each $g$, being continuous and real. Shisha and Mond in $[14,15]$ described the rate of convergence for $K_{m}(g, y)$ in terms of the moduli of continuity of $g$.

Our aim is to derive the convergence theorem and the order of convergence of operators $\mathbb{T}_{n}(f ; y)$ given by expression $(2.6)$.

Lemma 3.1 From the generating function of the Brenke type polynomials given by (2.1), we obtain

$$
\begin{aligned}
\sum_{k=0}^{\infty} p_{k}(n y)= & g(1) B(n y), \\
\sum_{k=0}^{\infty} k p_{k}(n y)= & g^{\prime}(1) B(n y)+n y g(1) B^{\prime}(n y), \\
\sum_{k=0}^{\infty} k^{2} p_{k}(n y)= & n^{2} y^{2} g(1) B^{\prime \prime}(n y)+\left(2 g^{\prime}(1)+g(1)\right) n y B^{\prime}(n y)+\left(g^{\prime \prime}(1)+g^{\prime}(1)\right) B(n y), \\
\sum_{k=0}^{\infty} k^{3} p_{k}(n y)= & n^{3} y^{3} g(1) B^{3}(n y)+\left(3 g^{\prime}(1)+3 g(1)\right) n^{2} y^{2} B^{\prime \prime}(n y) \\
& +\left(3 g^{\prime \prime}(1)+6 g^{\prime}(1)+g(1)\right) n y B^{\prime}(n y)+\left(g^{3}(1)+3 g^{\prime \prime}(1)+g^{\prime}(1)\right) B(n y), \\
\sum_{k=0}^{\infty} k^{4} p_{k}(n y)= & n^{4} y^{4} g(1) B^{4}(n y)+\left(4 g^{\prime}(1)+6 g(1)\right) n^{3} y^{3} B^{3}(n y) \\
& +\left(6 g^{\prime \prime}(1)+18 g^{\prime}(1)+7 g(1)\right) n^{2} y^{2} B^{\prime \prime}(n y)
\end{aligned}
$$




$$
\begin{aligned}
& +\left(4 g^{3}(1)+18 g^{\prime \prime}(1)+14 g^{\prime}(1)+g(1)\right) n y B^{\prime}(n y) \\
& +\left(g^{4}(1)+6 g^{3}(1)+7 g^{\prime \prime}(1)+g^{\prime}(1)\right) B(n y) .
\end{aligned}
$$

Lemma 3.2 For all $y \in[0, \infty)$, we have

$$
\begin{aligned}
\mathbb{T}_{n}\left(e_{0} ; y\right)= & 1, \\
\mathbb{T}_{n}\left(e_{1} ; y\right)= & y \frac{B^{\prime}(n y)}{B(n y)}+\frac{A_{0}}{n}, \\
\mathbb{T}_{n}\left(e_{2} ; y\right)= & \frac{1}{\digamma_{1}^{-}}\left[n^{2} y^{2} \frac{B^{\prime \prime}(n y)}{B(n y)}+n y \frac{B^{\prime}(n y)}{B(n y)} B_{0}+C_{0}\right], \\
\mathbb{T}_{n}\left(e_{3} ; y\right)= & \frac{1}{\digamma_{2}^{-}}\left[n^{3} y^{3} \frac{B^{3}(n y)}{B(n y)}+n^{2} y^{2} \frac{B^{\prime \prime}(n y)}{B(n y)} A_{1}+n y \frac{B^{\prime}(n y)}{B(n y)} A_{2}+A_{3}\right], \\
\mathbb{T}_{n}\left(e_{4} ; y\right)= & \frac{1}{\digamma_{3}^{-}}\left[n^{4} y^{4} \frac{B^{4}(n y)}{B(n y)}+n^{3} y^{3} \frac{B^{3}(n y)}{B(n y)} B_{1}\right. \\
& \left.+n^{2} y^{2} \frac{B^{\prime \prime}(n y)}{B(n y)} B_{2}+n y \frac{B^{\prime}(n y)}{B(n y)} B_{3}+B_{4}\right],
\end{aligned}
$$

where

$$
\begin{aligned}
& A_{0}=\frac{g^{\prime}(1)}{g(1)}, \quad B_{0}=\frac{2 g(1)+2 g^{\prime}(1)}{g(1)}, \quad C_{0}=\frac{g^{\prime \prime}(1)+2 g^{\prime}(1)}{g(1)} \\
& A_{1}=\frac{3 g^{\prime}(1)+6 g(1)}{g(1)}, \quad A_{2}=\frac{3 g^{\prime \prime}(1)+12 g^{\prime}(1)+5 g(1)}{g(1)}, \\
& A_{3}=\frac{g^{3}(1)+6 g^{\prime \prime}(1)+5 g^{\prime}(1)}{g(1)} \\
& B_{1}=\frac{4 g^{\prime}(1)+12 g(1)}{g(1)}, \quad B_{2}=\frac{6 g^{\prime \prime}(1)+36 g^{\prime}(1)+32 g(1)}{g(1)} \\
& B_{3}=\frac{4 g^{3}(1)+36 g^{\prime \prime}(1)+64 g^{\prime}(1)+17 g(1)}{g(1)}, \\
& B_{4}=\frac{g^{4}(1)+12 g^{3}(1)+32 g^{\prime \prime}(1)+17 g^{\prime}(1)}{g(1)},
\end{aligned}
$$

and

$$
\digamma_{n}^{-}=\prod_{i=0}^{n}(n-i) .
$$

Proof Putting $f(t)=1$ in operator equation (2.6), we have

$$
\begin{aligned}
\mathbb{T}_{n}\left(e_{0} ; y\right) & =\frac{1}{g(1) B(n y)} \sum_{k=0}^{\infty} \frac{p_{k}(n y)}{B(n+1, k)} \int_{0}^{\infty} \frac{t^{k-1}}{(1+t)^{n+k+1}} d t \\
& =\frac{1}{g(1) B(n y)} \frac{g(1) B(n y)}{B(n+1, k)} B(k, n+1) \\
& =1 .
\end{aligned}
$$


Now, putting $f(t)=t$ in operator equation (2.6), we have

$$
\begin{aligned}
\mathbb{T}_{n}\left(e_{1} ; y\right) & =\frac{1}{g(1) B(n y)} \sum_{k=0}^{\infty} \frac{p_{k}(n y)}{B(n+1, k)} \int_{0}^{\infty} \frac{t^{k}}{(1+t)^{n+k+1}} d t \\
& =\frac{1}{g(1) B(n y)} \sum_{k=0}^{\infty} \frac{p_{k}(n y)}{B(n+1, k)} B(n, k+1) \\
& =\frac{1}{g(1) B(n y)} \sum_{k=0}^{\infty} p_{k}(n y) \frac{k}{n} \\
& =\frac{1}{n g(1) B(n y)} \sum_{k=0}^{\infty} k p_{k}(n y) \\
& =y \frac{B^{\prime}(n y)}{B(n y)}+\frac{g^{\prime}(1)}{n g(1)} \\
& =y \frac{B^{\prime}(n y)}{B(n y)}+\frac{A_{0}}{n} .
\end{aligned}
$$

Now, putting $f(t)=t^{2}$ in operator equation (2.6), we have

$$
\begin{aligned}
\mathbb{T}_{n}\left(e_{2} ; y\right) & =\frac{1}{g(1) B(n y)} \sum_{k=0}^{\infty} \frac{p_{k}(n y)}{B(n+1, k)} \int_{0}^{\infty} \frac{t^{k+1}}{(1+t)^{n+k+1}} d t \\
& =\frac{1}{g(1) B(n y)} \sum_{k=0}^{\infty} \frac{p_{k}(n y)}{B(n+1, k)} B(n-1, k+2) \\
& =\frac{1}{g(1) B(n y)} \sum_{k=0}^{\infty} p_{k}(n y) \frac{k+k^{2}}{\digamma_{1}^{-}} \\
& =\frac{1}{\digamma_{1}^{-} g(1) B(n y)} \sum_{k=0}^{\infty} k p_{k}(n y)+k^{2} p_{k}(n y) \\
& =\frac{1}{\digamma_{1}^{-}}\left[n^{2} y^{2} \frac{B^{\prime \prime}(n y)}{B(n y)}+n y \frac{B^{\prime}(n y)}{B(n y)}\left(\frac{2 g(1)+2 g^{\prime}(1)}{g(1)}\right)+\frac{g^{\prime \prime}(1)+2 g^{\prime}(1)}{g(1)}\right] \\
& =\frac{1}{\digamma_{1}^{-}}\left[n^{2} y^{2} \frac{B^{\prime \prime}(n y)}{B(n y)}+n y \frac{B^{\prime}(n y)}{B(n y)} B_{0}+C_{0}\right] .
\end{aligned}
$$

Similarly, putting $f(t)=t^{3}$ in operator equation (2.6), we have

$$
\begin{aligned}
\mathbb{T}_{n}\left(e_{3} ; y\right) & =\frac{1}{g(1) B(n y)} \sum_{k=0}^{\infty} \frac{p_{k}(n y)}{B(n+1, k)} \int_{0}^{\infty} \frac{t^{k+2}}{(1+t)^{n+k+1}} d t \\
& =\frac{1}{g(1) B(n y)} \sum_{k=0}^{\infty} \frac{p_{k}(n y)}{B(n+1, k)} B(n-2, k+3) \\
& =\frac{1}{g(1) B(n y)} \sum_{k=0}^{\infty} p_{k}(n y) \frac{k+3 k^{2}+k^{3}}{\digamma_{2}^{-}} \\
& =\frac{1}{\digamma_{2}^{-} g(1) B(n y)} \sum_{k=0}^{\infty} k p_{k}(n y)+3 k^{2} p_{k}(n y)+k^{3} p_{k}(n y)
\end{aligned}
$$




$$
\begin{aligned}
= & \frac{1}{\digamma_{2}^{-}}\left[n^{3} y^{3} \frac{B^{3}(n y)}{B(n y)}+n^{2} y^{2} \frac{B^{\prime \prime}(n y)}{B(n y)}\left(\frac{3 g^{\prime}(1)+6 g(1)}{g(1)}\right)+n y \frac{B^{\prime}(n y)}{B(n y)}\right. \\
& \left.\times\left(\frac{3 g^{\prime \prime}(1)+12 g^{\prime}(1)+5 g(1)}{g(1)}\right)+\left(\frac{g^{3}(1)+6 g^{\prime \prime}(1)+5 g^{\prime}(1)}{g(1)}\right)\right] \\
= & \frac{1}{\digamma_{2}^{-}}\left[n^{3} y^{3} \frac{B^{3}(n y)}{B(n y)}+n^{2} y^{2} \frac{B^{\prime \prime}(n y)}{B(n y)} A_{1}+n y \frac{B^{\prime}(n y)}{B(n y)} A_{2}+A_{3}\right] .
\end{aligned}
$$

Again, putting $f(t)=t^{4}$ in operator equation (2.6), we have

$$
\begin{aligned}
\mathbb{T}_{n}\left(e_{4} ; y\right)= & \frac{1}{g(1) B(n y)} \sum_{k=0}^{\infty} \frac{p_{k}(n y)}{B(n+1, k)} \int_{0}^{\infty} \frac{t^{k+3}}{(1+t)^{n+k+1}} d t \\
= & \frac{1}{g(1) B(n y)} \sum_{k=0}^{\infty} \frac{p_{k}(n y)}{B(n+1, k)} B(n-3, k+4) \\
= & \frac{1}{g(1) B(n y)} \sum_{k=0}^{\infty} p_{k}(n y) \frac{3 k+7 k^{2}+6 k^{3}+k^{4}}{\digamma_{3}^{-}} \\
= & \frac{1}{\digamma_{3}^{-} g(1) B(n y)} \sum_{k=0}^{\infty} 3 k p_{k}(n y)+7 k^{2} p_{k}(n y)+6 k^{3} p_{k}(n y)+k^{4} p_{k}(n y) \\
= & \frac{1}{\digamma_{3}^{-}}\left[n^{4} y^{4} \frac{B^{4}(n y)}{B(n y)}+n^{3} y^{3} \frac{B^{3}(n y)}{B(n y)}\left(\frac{4 g^{\prime}(1)+12 g(1)}{g(1)}\right)+n^{2} y^{2} \frac{B^{\prime \prime}(n y)}{B(n y)}\right. \\
& \times\left(\frac{6 g^{\prime \prime}(1)+36 g^{\prime}(1)+32 g(1)}{g(1)}\right) \\
& +n y \frac{B^{\prime}(n y)}{B(n y)}\left(\frac{4 g^{3}(1)+36 g^{\prime \prime}(1)+64 g^{\prime}(1)+17 g(1)}{g(1)}\right) \\
& \left.+\left(\frac{g^{4}(1)+12 g^{3}(1)+32 g^{\prime \prime}(1)+17 g^{\prime}(1)}{g(1)}\right)\right] \\
= & \frac{1}{\digamma_{3}^{-}}\left[n^{4} y^{4} \frac{B^{4}(n y)}{B(n y)}+n^{3} y^{3} \frac{B^{3}(n y)}{B(n y)} B_{1}+n^{2} y^{2} \frac{B^{\prime \prime}(n y)}{B(n y)} B_{2}\right. \\
& \left.+n y \frac{B^{\prime}(n y)}{B(n y)} B_{3}+B_{4}\right] .
\end{aligned}
$$

Lemma 3.3 For $\mathbb{T}_{n}(f ; y)$ operators and for $y \in[0, \infty)$, the following identities are satisfied:

$$
\begin{aligned}
\mathbb{T}_{n}(s-y ; y)=( & \left.\frac{B^{\prime}(n y)-B(n y)}{B(n y)}\right) y+\frac{A_{0}}{n}, \\
\mathbb{T}_{n}\left((s-y)^{2} ; y\right)= & \left(\frac{\frac{n}{n-1} B^{\prime \prime}(n y)-2 B^{\prime}(n y)+B(n y)}{B(n y)}\right) y^{2} \\
& +\left(\frac{B^{\prime}(n y)}{B(n y)(n-1)} B_{0}-\frac{2}{n} A_{0}\right) y+\frac{C_{0}}{\digamma_{1}^{-}} \\
\mathbb{T}_{n}\left((s-y)^{4} ; y\right)= & \left(\frac{n^{4}}{\digamma_{3}^{-}} \frac{B^{4}(n y)}{B(n y)}-4 \frac{n^{3}}{\digamma_{2}^{-}} \frac{B^{3}(n y)}{B(n y)}+6 \frac{n^{2}}{\digamma_{1}^{-}} \frac{B^{\prime \prime}(n y)}{B(n y)}-4 \frac{B^{\prime}(n y)}{B(n y)}+1\right) y^{4} \\
& +\left(\frac{n^{3}}{\digamma_{3}^{-}} \frac{B^{3}(n y)}{B(n y)} B_{1}-4 \frac{n^{2}}{\digamma_{2}^{-}} \frac{B^{\prime \prime}(n y)}{B(n y)} A_{1}+6 \frac{n}{\digamma_{1}^{-}} \frac{B^{\prime}(n y)}{B(n y)} B_{0}-4 \frac{A_{0}}{n}\right) y^{3}
\end{aligned}
$$




$$
\begin{aligned}
& +\left(\frac{n^{2}}{\digamma_{3}^{-}} \frac{B^{\prime \prime}(n y)}{B(n y)} B_{2}-4 \frac{n}{\digamma_{2}^{-}} \frac{B^{\prime}(n y)}{B(n y)} A_{2}+6 \frac{C_{0}}{\digamma_{1}^{-}}\right) y^{2} \\
& +\left(\frac{n}{\digamma_{3}^{-}} \frac{B^{\prime}(n y)}{B(n y)} B_{3}-4 \frac{A_{3}}{\digamma_{2}^{-}}\right) y+\frac{B_{4}}{\digamma_{3}^{-}},
\end{aligned}
$$

where $A_{0}, B_{0}, C_{0}, A_{1}, A_{2}, A_{3}, B_{1}, B_{2}, B_{3}, B_{4}$, and $\digamma_{1}^{-}$are given by equations (3.6)-(3.10) respectively.

Proof In view of the linearity property of $\mathbb{T}_{n}$, it follows that

$$
\begin{aligned}
\mathbb{T}_{n}(s-y ; y)= & \mathbb{T}_{n}(s ; y)-y \mathbb{T}_{n}(1 ; y), \\
\mathbb{T}_{n}\left((s-y)^{2} ; y\right)= & \mathbb{T}_{n}\left(s^{2} ; y\right)-2 y \mathbb{T}_{n}(s ; y)+y^{2} \mathbb{T}_{n}(1 ; y), \\
\mathbb{T}_{n}\left((s-y)^{4} ; y\right)= & \mathbb{T}_{n}\left(s^{4} ; y\right)-4 y \mathbb{T}_{n}\left(s^{3} ; y\right)+6 y^{2} \mathbb{T}_{n}\left(s^{2} ; y\right) \\
& -4 y^{3} \mathbb{T}_{n}(s ; y)+y^{4} \mathbb{T}_{n}(1 ; y),
\end{aligned}
$$

which, on applying Lemma 3.2, yields assertions (3.17), (3.18), and (3.19), respectively.

\section{Theorem 3.1 Let}

$$
1=\lim _{y \rightarrow \infty} \frac{B^{\prime}(y)}{B(y)} \text { and } \quad 1=\lim _{y \rightarrow \infty} \frac{B^{\prime \prime}(y)}{B(y)} .
$$

Iff $\in C[0, \infty) \cap E$, then $\lim _{n \rightarrow \infty} \mathbb{T}_{n}(f ; y)=f(y)$ and the operators $\mathbb{T}_{n}$ in each compact subset of $[0, \infty)$ converge uniformly, where $E:=\left\{f:\right.$ for all $y \in[0, \infty),|f(y)| \leq c e^{A y}, A \in \mathbb{R}$ and $c \in$ $\left.\mathbb{R}^{+}\right\}$.

Proof By Lemma 3.2, we find

$$
\lim _{n \rightarrow \infty} \mathcal{T}_{n}\left(e_{j}, y\right)=y^{j}, \quad j=0,1,2
$$

The above convergence is verified uniformly on each compact subset of $[0, \infty)$. Applying Korovkin's theorem, the desired result is achieved.

\section{Order of convergence}

Now we recall the following definitions.

Definition 4.1 The II modulus of continuity of the function $h \in \mathbb{C}_{\mathbb{B}}[0, \infty)$ is defined by

$$
\mathbb{W}_{2}(g ; \delta):=\sup _{0<t \leq \delta}\|g(\cdot+2 k)-2 g(\cdot+k)+g(\cdot)\|_{\mathbb{C}_{\mathbb{B}}}
$$

where $\mathbb{C}_{\mathbb{B}}[0, \infty)$ is the class of real-valued functions defined on $[0, \infty)$, which are bounded and uniformly continuous with the norm

$$
\|g\|_{\mathbb{C}_{\mathbb{B}}}=\sup _{y \in[0, \infty)}|g(y)| .
$$


Definition 4.2 Peetre's $\mathbb{K}$-functional of the function $g \in \mathbb{C}_{\mathbb{B}}[0, \infty)$ is defined by

$$
\mathbb{K}(g ; \delta):=\inf _{h \in \mathbb{C}_{\mathbb{B}}^{2}[0, \infty)}\left\{\|g-h\|_{\mathbb{C}_{\mathbb{B}}}+\delta\|h\|_{\mathbb{C}_{\mathbb{B}}^{2}}\right\}
$$

where

$$
\mathbb{C}_{\mathbb{B}}^{2}[0, \infty):=\left\{h \in \mathbb{C}_{\mathbb{B}}[0, \infty): h^{\prime}, h^{\prime \prime} \in \mathbb{C}_{\mathbb{B}}[0, \infty)\right\}
$$

and the norm

$$
\|h\|_{\mathbb{C}_{\mathbb{B}}^{2}}:=\|h\|_{\mathbb{C}_{\mathbb{B}}}+\left\|h^{\prime}\right\|_{\mathbb{C}_{\mathbb{B}}}+\left\|h^{\prime \prime}\right\|_{\mathbb{C}_{\mathbb{B}}}(\text { see }[3])
$$

It is clear that the following inequality

$$
\mathbb{K}(g ; \delta) \leq M\left\{w_{2}(g, \delta)+\min (1, \delta)\|g\|_{\mathbb{C}_{\mathbb{B}}}\right\}
$$

holds for all $\delta>0$. The constant M is independent of $g$ and $\delta$.

Lemma 4.1 (Gavrea and Raşa [5]) Let $h \in \mathbb{C}^{2}[0, a]$ and $\left(\mathbb{K}_{m}\right)_{m \geq 0}$ be a sequence of positive linear operators with the property $\mathbb{K}_{m}(1 ; y)=1$. Then

$$
\left|\mathbb{K}_{m}(h ; y)-h(y)\right| \leq\left\|h^{\prime}\right\| \sqrt{\mathbb{K}_{m}\left((s-y)^{2} ; y\right)}+\frac{1}{2}\left\|h^{\prime \prime}\right\| \mathbb{K}_{m}\left((s-y)^{2} ; y\right) .
$$

Lemma 4.2 (Zhuk [18]) Let $g \in \mathbb{C}[a, b]$ and $h \in\left(0, \frac{a-b}{2}\right)$. Let $g_{h}$ be the second-order Steklov function attached to the function $g$. Then the following inequalities are satisfied:

$$
\begin{aligned}
& \text { (i) }\left\|g_{h}-g\right\| \leq \frac{3}{4} \mathbb{W}_{2}(g ; h), \\
& \text { (ii) }\left\|g_{h}^{\prime \prime}\right\| \leq \frac{3}{2 h^{2}} \mathbb{W}_{2}(g ; h) .
\end{aligned}
$$

Now, we compute the rates of convergence of the operators $\mathbb{T}_{n}(f ; y)$ to $f$ by means of a classical approach, the modulus of continuity, and Peetre's $\mathbb{K}$-functional. The following result gives the rates of convergence of the sequence $\mathbb{T}_{n}(f ; y)$ to $f$ by means of modulus of continuity.

Theorem 4.1 For $f \in C[0, a]$, the following inequality is satisfied:

$$
\left|\mathbb{T}_{n}(f ; y)-f(y)\right| \leq \frac{2}{a}\|f\| h^{2}+\frac{3}{4}\left(a+2+h^{2}\right) w_{2}(f ; h),
$$

where

$$
h:=h_{n}(y)=\sqrt[4]{\mathbb{T}_{n}\left((s-y)^{2} ; y\right)},
$$

and the second order modulus of continuity is given by $w_{2}(f ; \delta)$ with the norm $\|f\|=$ $\max _{y \in[a, b]}|f(y)|$ 
Proof Let $f_{h}$ be the second-order Steklov function attached to the function $f$. In view of identity (3.1), we have

$$
\begin{aligned}
\left|\mathbb{T}_{n}(f ; y)-f(y)\right| & \leq\left|\mathbb{T}_{n}\left(f-f_{h} ; y\right)\right|+\left|\mathbb{T}_{n}\left(f_{h} ; y\right)-f_{h}(y)\right|+\left|f_{h}(y)-f(y)\right|, \\
& \leq 2\left|f_{h}-f \|+\right| \mathbb{T}_{h}\left(f_{h} ; y\right)-f_{h}(y) \mid,
\end{aligned}
$$

which on using inequality (4.7) becomes

$$
\left|\mathbb{T}_{n}(f ; y)-f(y)\right| \leq \frac{3}{2} w_{2}(f ; h)+\left|\mathbb{T}_{n}\left(f_{h} ; y\right)-f_{h}(y)\right|
$$

Taking into account that $f_{h} \in C^{2}[0, a]$, from Lemma 4.1, it follows that

$$
\left|\mathbb{T}_{n}\left(f_{h} ; y\right)-f_{h}(y)\right| \leq\left\|f_{h}^{\prime}\right\| \sqrt{\mathbb{T}_{n}\left((s-y)^{2} ; y\right)}+\frac{1}{2}\left\|f_{h}^{\prime \prime}\right\| \mathbb{T}_{n}\left((s-y)^{2} ; y\right),
$$

which in view of inequality (4.8) becomes

$$
\left|\mathbb{T}_{n}\left(f_{h} ; y\right)-f_{h}(y)\right| \leq\left\|f_{h}^{\prime}\right\| \sqrt{\mathbb{T}_{n}\left((s-y)^{2} ; y\right)}+\frac{3}{4 h^{2}} w_{2}(f ; h) \mathbb{T}_{n}\left((s-y)^{2} ; y\right) .
$$

Further, the Landau inequality

$$
\left\|f_{h}^{\prime}\right\| \leq \frac{2}{\alpha}\left\|f_{h}\right\|+\frac{a}{2}\left\|f_{h}^{\prime \prime}\right\|
$$

combined with inequality (4.8) gives

$$
\left\|f_{h}^{\prime}\right\| \leq \frac{2}{a}\|f\|+\frac{3 a}{4 h^{2}} w_{2}(f ; h)
$$

Using inequality (4.14) in inequality (4.13) and taking $h=\sqrt[4]{\mathbb{T}_{n}\left((s-y)^{2}\right) ; y}$, we find

$$
\left|\mathbb{T}_{h}\left(f_{h} ; y\right)-f_{h}(y)\right| \leq \frac{2}{a}\|f\| h^{2}+\frac{3}{4}\left(a+h^{2}\right) w_{2}(f ; h) .
$$

Making use of inequality (4.15) in inequality (4.11) can lead to assertion (4.9).

Theorem 4.2 Let $f \in C_{B}^{2},[0, \infty)$, then

$$
\left|\mathbb{T}_{n}(f ; y)-f(y)\right| \leq \xi\|f\|_{C_{B}^{2}},
$$

where

$$
\begin{aligned}
\xi:= & \xi_{n}(y) \\
= & {\left[\left\{\frac{\frac{n}{n-1} B^{\prime \prime}(n y)-2 B^{\prime}(n y)+B(n y)}{2 B(n y)}\right\} y^{2}\right.} \\
& \left.+\left\{\left(1+\frac{B_{0}}{2(n-1)}\right) \frac{B^{\prime}(n y)}{B(n y)}-1-\frac{A_{0}}{n}\right\} y+\frac{C_{0}}{\digamma_{1}^{-}}\right] .
\end{aligned}
$$


Proof Using Taylor's expansion of $f$, the linearity property of the operator $\mathbb{T}_{n}$, and (3.1), it follows that

$$
\mathbb{T}_{n}(f ; y)-f(y)=f^{\prime}(y) \mathbb{T}_{n}(s-y ; y)+\frac{1}{2} f^{\prime \prime}(\eta) \mathbb{T}_{n}\left((s-y)^{2} ; y\right), \quad \eta \in(y, s) .
$$

From Lemma (3.2), it is evident that

$$
\mathbb{T}_{n}(s-y ; y)=\left(\frac{B^{\prime}(n y)}{B(n y)}-1\right) y+\frac{A_{0}}{n} \geq 0
$$

for $s \geq y$, thus by considering Lemma (3.2) and (3.3) in (4.17), we can write

$$
\begin{aligned}
\left|\mathbb{T}_{n}(f ; y)-f(y)\right| \leq & \left\{\left(\frac{B^{\prime}(n y)}{B(n y)}-1\right) y+\frac{A_{0}}{n}\right\}\left\|f^{\prime}\right\|_{C_{B}} \\
& +\frac{1}{2}\left\{\left(\frac{\frac{n}{n-1} B^{\prime \prime}(n y)-2 B^{\prime}(n y)+B(n y)}{B(n y)}\right) y^{2}\right. \\
& \left.+\left(\frac{B^{\prime}(n y)}{B(n y)(n-1)} B_{0}-\frac{2}{n} A_{0}\right) y+\frac{C_{0}}{\digamma_{1}^{-}}\right\}\left\|f^{\prime \prime}\right\|_{C_{B}} \\
\leq & {\left[\left\{\frac{\frac{n}{n-1} B^{\prime \prime}(n y)-2 B^{\prime}(n y)+B(n y)}{2 B(n y)}\right\} y^{2}\right.} \\
& \left.+\left\{\left(1+\frac{B_{0}}{2(n-1)}\right) \frac{B^{\prime}(n y)}{B(n y)}-1-\frac{A_{0}}{n}\right\} y+\frac{C_{0}}{\digamma_{1}^{-}}\right]\|f\|_{C_{B}^{2},}
\end{aligned}
$$

which completes the proof.

Theorem 4.3 Iff $\in C_{B}[0, \infty)$, then one has

$$
\left|\mathbb{T}_{n}(f ; y)-f(y)\right| \leq 2 M\left\{w_{2}(f ; \sqrt{\delta})+\min (1, \delta)\|f\|_{C_{B}}\right\},
$$

where

$$
\delta:=\delta_{n}(y)=\frac{1}{2} \xi_{n}(y)
$$

and $M \geq 0$ is a constant, which is independent of the functions $f$ and $\delta . A l s o, \xi_{n}(y)$ is the same as in Theorem 4.2 .

Proof Suppose that $g \in C_{B}^{2}[0, \infty)$, from previous Theorem 4.2, we have

$$
\begin{aligned}
\left|\mathbb{T}_{n}(f ; y)-f(y)\right| & \leq\left|\mathbb{T}_{n}(f-g ; y)\right|+\left|\mathbb{T}_{n}(g ; y)-g(y)\right|+|g(y)-f(y)| \\
& \leq 2\|f-g\|_{C_{B}}+\xi\|g\|_{C_{B}^{2}}=2\left[\|f-g\|_{C_{B}}+\delta\|g\|_{C_{B}^{2}}\right] .
\end{aligned}
$$

Since the l.h.s of the above inequality does not depend on the function $g \in C_{B}^{2}[0, \infty)$,

$$
\left|\mathbb{T}_{n}(f ; y)-f(y)\right| \leq 2 K(f ; \delta),
$$

where K is Peetre's functional defined by (4.3). By using relation (4.5) in (4.22), the inequality

$$
\left|\mathbb{T}_{n}(f ; y)-f(y)\right| \leq 2 M\left\{w_{2}(f ; \sqrt{\delta})+\min (1, \delta)\|f\|_{C_{B}}\right\}
$$

holds. 


\section{Weighted approximation}

Here, some properties of approximation for the operator $\mathbb{T}_{n}$ of a space of weighted continuous functions are given, for which the succeeding class of functions is defined on $[0, \infty)$.

Consider $B_{y^{2}}[0, \infty)$ defined on $[0, \infty)$ as the set of all functions $h$ which satisfies $|h(y)| \leq$ $M_{h}\left(1+y^{2}\right)$, where $M_{h}$ depends on $h$ is a constant. Also, consider $C_{y^{2}}[0, \infty)$ as the subspace of $B_{y}^{2}[0, \infty)$ of all continuous functions. Further, $C_{y^{2}}^{*}[0, \infty)$ as the subspace of $h \in C_{y^{2}}[0, \infty)$ for which $\lim _{|y| \rightarrow \infty} \frac{h(y)}{1+y^{2}}$ is finite. It is evident that $C_{y^{2}}^{*}[0, \infty) \subset C_{y^{2}}[0, \infty) \subset B_{y}^{2}[0, \infty)$. The norm on $C_{y^{2}}^{*}[0, \infty)$ is given as follows:

$$
\|h\|_{y^{2}}=\sup _{y \in[0, \infty)} \frac{|h(y)|}{1+y^{2}} .
$$

Lemma 5.1 Let the weight function $\rho(y)=1+y^{2}$. If $h \in C_{y^{2}}[0, \infty)$, then

$$
\left\|\mathbb{T}_{n}(\rho ; y)\right\|_{y^{2}} \leq 1+M
$$

Proof By expressions (3.1) and (3.3) of Lemma 3.2, for $n>1$, we get

$$
\mathbb{T}_{n}(\rho ; y)=1+\frac{n}{n-1} \frac{B^{\prime \prime}(n y)}{B(n y)} y^{2}+\frac{B_{0}}{n-1} \frac{B^{\prime}(n y)}{B(n y)} y+\frac{C_{0}}{n(n-1)} .
$$

Then we deduce

$$
\begin{aligned}
\left\|\mathbb{T}_{n}(\rho ; y)\right\|_{y^{2}}= & \sup _{y \geq 0}\left\{\frac{1}{1+y^{2}}+\frac{n}{\left(1+y^{2}\right)(n-1)} \frac{B^{\prime \prime}(n y)}{B(n y)} y^{2}+\frac{B_{0}}{\left(1+y^{2}\right)(n-1)} \frac{B^{\prime}(n y)}{B(n y)} y\right. \\
& \left.+\frac{C_{0}}{n\left(1+y^{2}\right)(n-1)}\right\} \\
\leq & \sup _{y \geq 0}\left\{1+\frac{n}{(n-1)} \frac{B^{\prime \prime}(n y)}{B(n y)}+\frac{B_{0}}{(n-1)} \frac{B^{\prime}(n y)}{B(n y)}+\frac{C_{0}}{n(n-1)}\right\} .
\end{aligned}
$$

As we know already,

$$
\lim _{n \rightarrow \infty} \frac{B^{\prime}(n y)}{B(n y)}=1, \quad \lim _{n \rightarrow \infty} \frac{B^{\prime \prime}(n y)}{B(n y)}=1,
$$

also, we know that

$$
\lim _{n \rightarrow \infty} \frac{n}{n-1}=1, \quad \lim _{n \rightarrow \infty} \frac{1}{n-1}=0 \quad \text { and } \quad \lim _{n \rightarrow \infty} \frac{1}{n^{2}-n}=0,
$$

thus in view of these assumptions there exists a positive constant $M$ such that

$$
\left\|\mathbb{T}_{n}(\rho ; y)\right\|_{y^{2}} \leq 1+M
$$

which concludes the proof. 
By using Lemma 5.1, one can see that the operator $\mathbb{T}_{n}$ defined by (2.6) acts from $C_{y^{2}}[0, \infty)$ to $B_{y^{2}}[0, \infty)$.

Theorem 5.1 Lthe5.1 Let $\mathbb{T}_{n}$ be the sequence of positive linear operators defined by (2.6)

and $\rho(y)=1+y^{2}$ be a weight function, then for each $f \in C_{y^{2}}^{*}[0, \infty)$,

$$
\lim _{n \rightarrow \infty}\left\|\mathbb{T}_{n}(f ; y)-f(y)\right\|_{y^{2}}=0
$$

Proof By using the weighted Korovkin theorem presented by Gadzhiev [4], it is enough to verify the following conditions:

$$
\left\|\mathbb{T}_{n}(1 ; y)-1\right\|_{y^{2}}=0
$$

By equation (3.2), we have

$$
\begin{aligned}
\left\|\mathbb{T}_{n}\left(e_{1} ; y\right)-e_{1}(y)\right\|_{y^{2}} & =\sup _{y \geq 0}\left|\frac{B^{\prime}(n y)}{B(n y)} \frac{y}{1+y^{2}}+\frac{A_{0}}{n} \frac{1}{1+y^{2}}-\frac{y}{1+y^{2}}\right| \\
& =\sup _{y \geq 0}\left|\left(\frac{B^{\prime}(n y)}{B(n y)}-1\right) \frac{y}{1+y^{2}}+\frac{A_{0}}{n} \frac{1}{1+y^{2}}\right| \\
& =\left(\frac{B^{\prime}(n y)}{B(n y)}-1\right)+\frac{A_{0}}{n},
\end{aligned}
$$

therefore, keeping in view the fact $\lim _{n \rightarrow \infty} \frac{B^{\prime}(n y)}{B(n y)}=1$, we get

$$
\lim _{n \rightarrow \infty}\left\|\mathbb{T}_{n}\left(e_{1} ; y\right)-e_{1}(y)\right\|_{y^{2}}=0
$$

By equation (3.3), we have

$$
\begin{aligned}
\left\|\mathbb{T}_{n}\left(e_{2} ; y\right)-e_{2}(y)\right\|_{y^{2}}= & \sup _{y \geq 0} \mid\left(\frac{n}{n-1} \frac{B^{\prime \prime}(n y)}{B(n y)}-1\right) \frac{y}{1+y^{2}} \\
& +\frac{B_{0}}{n-1} \frac{B^{\prime}(n y)}{B(n y)} \frac{y}{1+y^{2}}+\frac{C_{0}}{n(n-1)\left(1+y^{2}\right)} \mid \\
\leq & \left(\frac{n}{n-1} \frac{B^{\prime \prime}(n y)}{B(n y)}-1\right)+\frac{B_{0}}{n-1} \frac{B^{\prime}(n y)}{B(n y)}+\frac{C_{0}}{n(n-1)},
\end{aligned}
$$

therefore, keeping in view the fact $\lim _{n \rightarrow \infty} \frac{B^{\prime \prime}(n y)}{B(n y)}=1$ and $\lim _{n \rightarrow \infty} \frac{n}{n-1}=1$, we get

$$
\lim _{n \rightarrow \infty}\left\|\mathbb{T}_{n}\left(e_{2} ; y\right)-e_{2}(y)\right\|_{y^{2}}=0
$$

Hence the proof is completed.

\section{Special cases of the operators $\mathbb{T}_{n}$ and further properties}

The Gould-Hopper polynomials $p_{k}^{d+1}(y ; h)$ given by the identity

$$
p_{k}^{d+1}(y ; h)=\sum_{m=0}^{[k / d+1]} \frac{k !}{m !(k-(d+1) m) !} h^{m} y^{k-(d+1) m}
$$


possess the generating expression

$$
e^{h t^{d+1}} \exp (y t)=\sum_{k=0}^{\infty} p_{k}^{d+1}(y, h) \frac{t^{k}}{k !}
$$

These $p_{k}^{d+1}(y ; h)$ polynomials are $p_{k}(y)$ Brenke polynomials for $g(t)=e^{h t^{d+1}}$ and $B(t)=e^{t}$ in expression (2.1). Thus, for $g(t)=e^{h t^{d+1}}$ and $B(t)=e^{t}$ in equation (2.6) gives the following Durrmeyer type Jakimovski-Leviatan operators $\mathbb{T}_{n}^{*}(f ; y)$ involving the $p_{k}^{d+1}(y ; h)$ polynomials:

$$
\mathbb{T}_{n}^{*}(f ; y):=e^{-n y-h} \sum_{k=0}^{\infty} \frac{p_{k}^{d+1}(n y, h)}{B(n+1, k) k !} \int_{0}^{\infty} \frac{t^{k-1}}{(1+t)^{n+k+1}} f(t) d t
$$

beneath the presumption $h \geq 0$.

Now, to prove the Voronovskaya theorem for operators (6.3), first we prove the succeeding results.

Lemma 6.1 $\forall y \in[0, \infty)$, it follows that

$$
\begin{aligned}
\mathbb{T}_{n}^{*}\left(e_{0} ; y\right)= & 1 \\
\mathbb{T}_{n}^{*}\left(e_{1} ; y\right)= & y+\frac{1}{n} h(d+1), \\
\mathbb{T}_{n}^{*}\left(e_{2} ; y\right)= & \frac{1}{\digamma_{1}^{-}}\left[n^{2} y^{2}+n y(2+2 h(d+1))+h(d+1)(d+h(d+1)+2)\right], \\
\mathbb{T}_{n}^{*}\left(e_{3} ; y\right)= & \frac{1}{\digamma_{2}^{-}}\left[n^{3} y^{3}+n^{2} y^{2}(6+3 h(d+1))+n y(h(d+1)(3 d+3 h(d+1)+12)+5)\right. \\
& \left.+h(d+1)\left(3 h d^{2}+d^{2}+11 h d-d+6 h+5\right)\right], \\
\mathbb{T}_{n}^{*}\left(e_{4} ; y\right)= & \frac{1}{\digamma_{3}^{-}}\left[n^{4} y^{4}+n^{3} y^{3}(12+4 h(d+1))+n^{2} y^{2}(6 h(d+1)(h(d+1)+d+6)+32)\right. \\
& +n y\left(4 h(d+1)\left(d^{2}+8 d+3 h d^{2}+14 h d+9 h+16\right)+17\right) \\
& +\left(h(d+1)\left(d^{3}+9 d^{2}+22 d+24 h+17\right)\right. \\
& \left.\left.+h d(d+1)^{2}\left(6 d+9 h^{2} d+h^{2}+h d+2 h^{3} d+2 h^{3}+35 h-3\right)\right)\right] .
\end{aligned}
$$

\section{Lemma 6.2}

$$
\begin{aligned}
& \mathbb{T}_{n}^{*}((s-y) ; y)=\frac{1}{n} h(d+1), \\
& \mathbb{T}_{n}^{*}\left((s-y)^{2} ; y\right)=\frac{y^{2}+2 y}{n-1}+\frac{2 h(d+1)}{n(n-1)} y+\frac{1}{\digamma_{1}^{-}}[h(d+1)(d+h(d+1)+2)]
\end{aligned}
$$

and

$$
\begin{aligned}
\mathbb{T}_{n}^{*}\left((s-y)^{4} ; y\right)= & \left\{\frac{n^{4}}{\digamma_{3}^{-}}-4 \frac{n^{3}}{\digamma_{2}^{-}}+6 \frac{n^{2}}{\digamma_{1}^{-}}-3\right\} y^{4}+\left\{\frac{n^{3}}{\digamma_{3}^{-}}(4 h(d+1)+12)\right. \\
& \left.-4 \frac{n^{2}}{\digamma_{2}^{-}}(3 h(d+1)+6)+6 \frac{n}{\digamma_{1}^{-}}(2 h(d+1)+2)-\frac{4}{\digamma_{0}^{-}} h(d+1)\right\} y^{3}
\end{aligned}
$$




$$
\begin{aligned}
& +\left\{\frac{n^{2}}{\digamma_{3}^{-}}(6 h(d+1)(h(d+1)+d+6)+32)-4 \frac{n}{\digamma_{2}^{-}}(h(d+1)\right. \\
& \left.\times(3 d+3 h(d+1)+12)+5)+\frac{6}{\digamma_{1}^{-}} h(d+1)(d+h(d+1)+2)\right\} y^{2} \\
& +\left\{\frac{n}{\digamma_{3}^{-}}\left(4 h(d+1)\left(d^{2}+8 d+3 h d^{2}+14 h d+9 h+16\right)+17\right)\right. \\
& \left.-\frac{4}{\digamma_{2}^{-}}\left(h(d+1)\left(3 h d^{2}+d^{2}+11 h d-d+6 h+5\right)\right)\right\} y \\
& +\frac{1}{\digamma_{3}^{-}}\left\{\left(h(d+1)\left(d^{3}+9 d^{2}+22 d+24 h+17\right)\right.\right. \\
& \left.\left.+h d(d+1)^{2}\left(6 d+9 h^{2} d+h^{2}+h d+2 h^{3} d+2 h^{3}+35 h-3\right)\right)\right\} .
\end{aligned}
$$

Lemma 6.3 Here, we possess the limits:

(i) $\quad \lim _{n \rightarrow \infty} n \mathbb{T}_{n}^{*}\left((s-y)^{2} ; y\right)=y^{2}+2 y$

and

$$
\text { (ii) } \lim _{n \rightarrow \infty} n^{2} \mathbb{T}_{n}^{*}\left((s-y)^{4} ; y\right)=3 y^{4}+12 y^{3}+12 y^{2} \text {. }
$$

Proof In view of equation (6.9), we have

$$
\begin{aligned}
\lim _{n \rightarrow \infty} n \mathbb{T}_{n}^{*}\left((s-y)^{2} ; y\right) & =n\left\{\frac{y^{2}+2 y}{n-1}+\frac{2 h(d+1)}{n(n-1)} y+\frac{1}{\digamma_{1}^{-}}[h(d+1)(d+h(d+1)+2)]\right\} \\
& =y^{2}+2 y,
\end{aligned}
$$

where $\lim _{n \rightarrow \infty} \frac{n}{n-1}=1$ and $\lim _{n \rightarrow \infty} \frac{1}{n-1}=0$, and in view of equation (6.10), we have

$$
\begin{aligned}
& \lim _{n \rightarrow \infty} n^{2}\left\{\frac{n^{4}}{\digamma_{3}^{-}}-4 \frac{n^{3}}{\digamma_{2}^{-}}+6 \frac{n^{2}}{\digamma_{1}^{-}}-3\right\} y^{4}=\lim _{n \rightarrow \infty} n^{2}\left\{\frac{3 n+18}{(n-1)(n-2)(n-3)}\right\}=3, \\
& \lim _{n \rightarrow \infty} n^{2}\left\{\frac{n^{3}}{\digamma_{3}^{-}}(4 h(d+1)+12)-4 \frac{n^{2}}{\digamma_{2}^{-}}(3 h(d+1)+6)+6 \frac{n}{\digamma_{1}^{-}}(2 h(d+1)+2)\right. \\
& \left.\quad-\frac{4}{\digamma_{0}^{-}} h(d+1)\right\}=12, \\
& \lim _{n \rightarrow \infty} n^{2}\left\{\frac{n^{2}}{\digamma_{3}^{-}}(6 h(d+1)(h(d+1)+d+6)+32)-4 \frac{n}{\digamma_{2}^{-}}(h(d+1)\right. \\
& \left.\quad \times(3 d+3 h(d+1)+12)+5)+\frac{6}{\digamma_{1}^{-}} h(d+1)(d+h(d+1)+2)\right\}=12, \\
& \lim _{n \rightarrow \infty} n^{2}\left\{\frac{n}{\digamma_{3}^{-}}\left(4 h(d+1)\left(d^{2}+8 d+3 h d^{2}+14 h d+9 h+16\right)+17\right)-\frac{4}{\digamma_{2}^{-}}(h(d+1)\right. \\
& \left.\left.\quad \times\left(3 h d^{2}+d^{2}+11 h d-d+6 h+5\right)\right)\right\}=0
\end{aligned}
$$


and

$$
\begin{aligned}
& \lim _{n \rightarrow \infty} n^{2} \frac{1}{\digamma_{3}^{-}}\left\{h(d+1)\left(d^{3}+9 d^{2}+22 d+24 h+17\right)\right. \\
& \left.\quad+h d(d+1)^{2}\left(6 d+9 h^{2} d+h^{2}+h d+2 h^{3} d+2 h^{3}+35 h-3\right)\right\}=0 .
\end{aligned}
$$

Theorem 6.1 For any $f \in C_{y^{2}}^{*}[0, \infty)$ such that $f^{\prime}, f^{\prime \prime} \in C_{y^{2}}^{*}[0, \infty)$, it follows that

$$
\lim _{n \rightarrow \infty} n\left(\mathbb{T}_{n}^{*}(f ; y)-f(y)\right)=h(d+1) f^{\prime}(y)+\frac{y^{2}+2 y}{2} f^{\prime \prime}(y)
$$

Proof Using Taylor's expansion of $f$, we obtain

$$
f(s)=f(y)+(s-y) f^{\prime}(y)+\frac{1}{2}(s-y)^{2} f^{\prime \prime}(y)+\eta(s, y)(s-y)^{2},
$$

where

$$
\eta(s, y) \rightarrow 0 \quad \text { as } s \rightarrow y \text {. }
$$

By the linearity of the operator $\mathbb{T}_{n}^{*}(f ; y)$, we get

$$
\begin{aligned}
& \mathbb{T}_{n}^{*}(f ; y)-f(y) \\
& \quad=\mathbb{T}_{n}^{*}(s-y ; y) f^{\prime}(y)+\frac{1}{2} \mathbb{T}_{n}^{*}\left((s-y)^{2} ; y\right) f^{\prime \prime}(y)+\mathbb{T}_{n}^{*}\left(\eta(s, y)\left((s-y)^{2} ; y\right)\right) .
\end{aligned}
$$

From Lemma 6.2, we have

$$
\begin{aligned}
\mathbb{T}_{n}^{*}(f ; y)-f(y)= & \left(\frac{1}{n} h(d+1)\right) f^{\prime}(y)+\frac{1}{2} f^{\prime \prime}(y)\left\{\frac{y^{2}+2 y}{n-1}+\frac{2 h(d+1)}{n(n-1)} y\right. \\
& \left.+\frac{1}{\digamma_{1}^{-}}[h(d+1)(d+h(d+1))+2]\right\}+\mathbb{T}_{n}^{*}\left(\eta(s, y)\left((s-y)^{2} ; y\right)\right) .
\end{aligned}
$$

For the last term of equation (6.14) or (6.15), using the Cauchy-Schwarz inequality, we get

$$
\lim _{n \rightarrow \infty} n\left(\eta(s, y)(s-y)^{2} ; y\right) \leq \sqrt{\lim _{n \rightarrow \infty}\left(\eta(s, y)\left(s^{2} ; y\right)\right)} \sqrt{\lim _{n \rightarrow \infty} n^{2}\left(\eta(s, y)\left((s-y)^{4} ; y\right)\right)} .
$$

Because of $\lim _{n \rightarrow \infty} \mathbb{T}_{n}^{*}\left(\eta^{2}((s, y) ; y)\right)=0$ and by Lemma 6.3(ii), $\lim _{n \rightarrow \infty} n^{2} \mathbb{T}_{n}^{*}\left(\left((s-y)^{4} ; y\right)\right)$ is finite, we have $\lim _{n \rightarrow \infty} n \mathbb{T}_{n}^{*}\left(\eta(s, y)\left((s-y)^{2} ; y\right)\right)=0$. Therefore we obtain

$$
\begin{aligned}
\lim _{n \rightarrow \infty} n\left(\mathbb{T}_{n}^{*}(f ; y)-f(y)\right)= & h(d+1) f^{\prime}(y)+\frac{1}{2} f^{\prime \prime}(y) \lim _{n \rightarrow \infty}\left\{\frac{n\left(y^{2}+2 y\right)}{n-1}+\frac{2 h(d+1)}{(n-1)} y\right. \\
& \left.+\frac{1}{n-1}[h(d+1)(d+h(d+1))+2]\right\} \\
= & h(d+1) f^{\prime}(y)+\frac{y^{2}+2 y}{2} f^{\prime \prime}(y) .
\end{aligned}
$$




\section{Funding}

None.

\section{Availability of data and materials}

Not applicable.

\section{Competing interests}

The authors declare that they have no competing interests.

Authors' contributions

All the authors contributed equally and they read and approved the final manuscript for publication.

\section{Author details}

'Department of CSE, North Campus, University of Kashmir, Srinagar, India. ${ }^{2}$ Department of Medical Research, China Medical University Hospital, China Medical University (Taiwan), Taichung, Taiwan. ${ }^{3}$ Al-Qaryah, Doharra, Street No. 1 (West), Aligarh, UP, 202002, India. ${ }^{4}$ Department of Mathematics, College of Arts and Sciences, Prince Sattam bin Abdulaziz University, Wadi Aldawser, 11991, Saudi Arabia.

\section{Publisher's Note}

Springer Nature remains neutral with regard to jurisdictional claims in published maps and institutional affiliations.

Received: 7 September 2020 Accepted: 2 June 2021 Published online: 12 June 2021

\section{References}

1. Alotaibi, A., Mursaleen, M.: Approximation of Jakimovski-Leviatan-Beta type integral operators via q-calculus. AIMS Math. 5(4), 3019-3034 (2020)

2. Chihara, T.S.: An Introduction to Orthogonal Polynomials. Gordon \& Breach, New York (1978)

3. Ditzian, Z., Totik, V.: Moduli of Smoothness. Springer Series in Computational Mathematics, vol. 9. Springer, New York (1987)

4. Gadzhiev, A.D.: The convergence problem for a sequence of positive linear operators on unbounded sets and theorems analogous to that of P. P. Krovokin. Sov. Math. Dokl. 15(5), 1433-1436 (1974)

5. Gavrea, I., Raşa, I.: Remarks on some quantitative Korovkin-type results. Rev. Anal. Numér. Théor. Approx. 22(2), 173-176 (1993)

6. Içöz, G., Tasdelen Yesildal, F., Dog̈ru, O.: Kantrovich process of linear positive operators via biorthogonal polynomials. J. Inequal. Spec. Funct. 3, 77-84 (2012)

7. İz, G., Varma, S., Sucu, S.: Approximation by operators including generalized Appell polynomials. Filomat 30(2), 429-440 (2016)

8. Jakimovski, A., Leviatan, D.: Generalized Szász operators for the approximation in the infinite interval. Mathematica 11, 97-103 (1969)

9. Karaisa, A.: Approximation by Durrmeyer type Jakimovski-Leviatan operators. Math. Methods Appl. Sci. 39(9), 2401-2410 (2015). https://doi.org/10.1002/mma.3650

10. Kilicman, A., Ayman Mursaleen, M., Al-Abied, A.A.H.: Stancu type Baskakov-Durrmeyer operators and approximation properties. Mathematics 8, Article ID 1164 (2020). https://doi.org/10.3390/math8071164

11. Korovkin, P.P.: On convergence of linear positive operators in the space of continuous functions. Dokl. Akad. Nauk SSSR $90,961-964(1953)$

12. Korovkin, P.P.: Linear Operators and Approximation Theory, Chapters I, II. Hindustan Publ., Delhi (1960) (Russian, 1959) English translation

13. Milovanovic, G.V., Mursaleen, M., Nasiruzzaman, M.: Modified Stancu type Dunkl generalization of Szász-Kantorovich operators. Rev. R. Acad. Cienc. Exactas Fís. Nat., Ser. A Mat. 112(1), 135-151 (2018)

14. Shisha, O., Mond, B.: The degree of convergence of linear positive operators. Proc. Natl. Acad. Sci. USA 60, 1196-1200 (1968)

15. Shisha, O., Mond, B.: The degree of approximation to periodic functions by linear positive operators. J. Approx. Theory 1, 335-339 (1968)

16. Szasz, O:: Generalization of S. Bernstein's polynomials to the infinite interval. J. Res. Natl. Bur. Stand. 45, $239-245$ (1950)

17. Varma, S., Sucu, S., İz, G.: Generalization of Szász operators involving Brenke type polynomials. Comput. Math. Appl. 64, 121-127 (2012)

18. Zhuk, V.V.: Functions of the Lip 1 class and S. N. Bernstein's polynomials. Vestn. Leningr. Univ., Mat. Meh. Astron. 1, 25-30 (1989) 